Revista Brasileira de Cartografia

ISSN 1808-0936 | https://doi.org/10.14393/revbrascartogr

Sociedade Brasileira de Cartografia, Geodésia, Fotogrametria e Sensoriamento Remoto

\title{
Análise de técnicas de detecção de mudança para mapeamento de desastres com uso de dados de sensoriamento remoto
}

\section{Analysis of change detection techniques for disaster mapping using remote sensing data}

\author{
Lucas Valerio de Oliveira ${ }^{1}$, Rogério Galante Negri ${ }^{2}$ e Leonardo Bacelar Lima Santos ${ }^{3}$
}

1 Unesp - Universidade Estadual Paulista "Júlio de Mesquita Filho", Departamento de Engenharia Ambiental,

São José dos Campos, Brasil. lucasvalerio@live.com

ORCID: https://orcid.org/0000-0002-1926-2938

2 Unesp - Universidade Estadual Paulista "Júlio de Mesquita Filho", Departamento de Engenharia Ambiental,

São José dos Campos, Brasil. rogerio.negri@ unesp.br

ORCID: https://orcid.org/0000-0002-4808-2362

3 Cemaden - Centro Nacional de Monitoramento e Alertas de Desastres Naturais,

São José dos Campos, Brasil. santoslbl@gmail.com

ORCID: https://orcid.org/0000-0002-3129-772X

Recebido: 11.2019 | Aceito: 02.2020

\begin{abstract}
Resumo: Nos anos recentes, o Brasil tem sofrido uma série de desastres ambientais, que por sua vez afetam a sociedade, economia e biodiversidade em níveis imensuráveis. O uso de dados obtidos por sensoriamento remoto surge como fonte de informação indispensável na realização de análises sobre os impactos causados pelos desastres ambientais. Dentre diferentes métodos propostos na literatura para o processamento e extração de informação das imagens de sensoriamento remoto, encontram-se as técnicas de detecção de mudança. De modo geral, tais técnicas permitem a obtenção de mapas capazes de expressar as alterações ocorridas na cobertura do solo de uma dada região e período. O uso destas técnicas aponta potencial quanto a geração de insumos para análises relacionadas aos impactos causados por desastres ambientais. Neste contexto, este estudo apresenta a aplicação e comparação entre diferentes métodos de detecção de mudança sobre áreas afetadas por recentes desastres ambientais no Brasil. Os métodos abordados neste trabalho são restritos ao conceito não supervisionado, os quais não exigem informações a priori sobre os dados. Foram realizadas três aplicações, relacionadas aos colapsos ocorridos nas barragens de Mariana e Brumadinho, no estado de Minas Gerais, e queimadas no estado do Mato Grosso, com uso de dados obtidos pelos satélites Landsat-8 e Sentinel-1 e 2. Os resultados obtidos evidenciam que tais técnicas, desde que parametrizadas adequadamente e fazendo uso de atributos favoráveis, são capazes de realizar mapeamentos sobre alterações derivadas dos eventos de desastres.
\end{abstract}

Palavras-chave: Detecção de Mudanças. Não-supervisionado. Desastres Ambientais.

\begin{abstract}
In recent years Brazil has suffered a series of environmental disasters that impaired its society, economy, and biodiversity in immeasurable levels. The use of remote sensing data rises as a fundamental source when analyzing environmental disaster impacts. Among several methods proposed in the literature, change detection techniques have shown potential use on environmental disaster investigations. In this context, this study presents an analysis with different change detection techniques applied to map the landscape modifications caused by recent environmental disasters in Brazil. The methods discussed in this paper are restricted to the unsupervised approach, which does not require a priori information about the data. Three applications were carried regarding the collapse that occurred in Mariana and Brumadinho dams, the state of Minas Gerais, and forest burning in the state of Mato Grosso. Images acquired by Landsat- 8 and Sentinel- 1 and 2 satellites were used in these studies. The results evidenced that such techniques, as long as parameterized and using favorable attributes, they can provide accurate maps regarding the changes from disaster events.
\end{abstract}

Keywords: Change detection. Unsupervised. Environmental disaster.

\section{INTRODUÇÃO}

Os desafios impostos pelos desastres, em suas diferentes formas, são severos e para enfrentá-los é preciso que as comunidades estejam preparadas com os devidos entendimentos sobre suas causas e formas de gerenciá-los (ADIKARAM; NAWARATHNA, 2018). Temas como: exposição ao risco e a vulnerabilidade 
social são amplamente discutidos e se tornaram conceitos fundamentais para servir de apoio durante o processo de gestão de desastres, que tem por fim o objetivo de mitigar os riscos ou atenuar os possíveis danos causados pelos eventos.

Os desastres por causas naturais são fenômenos físicos causados por eventos de início rápido ou lento que podem ser geofísicos (terremotos, deslizamentos de terra, tsunamis e atividade vulcânica), hidrológicos (avalanches e inundações), climatológicos (temperaturas extremas, secas e incêndios), meteorológicos (ciclones e tempestades) ou biológicos (epidemias de doenças e pragas de insetos ou animais) (HIDALGO; BAEZ, 2019). Segundo Tominaga (2015), os principais fenômenos relacionados aos desastres naturais que ocorrem no Brasil são derivados da dinâmica da Terra, eventos hidrológicos e climatológicos.

Desastres tecnológicos abrange uma segunda classe de desastres, tendo potencial de causar danos severos ao meio ambiente e à sociedade. Segundo Wanderley (2016), esse tipo de desastre está atribuído a falha de um sistema humano e a inúmeros fatores de erros e negligência dos procedimentos técnicos que por fim acabam resultando em danos (ou ferimentos) significativos ou mortes.

A utilização de Sensoriamento Remoto surge como uma excelente ferramenta no estudo e análise de desastres ambientais, uma vez que o processo de mensuração e de observação não depende de contato direto com o objeto de estudo (POURSANIDIS; CHRYSOULAKIS, 2017). O constante desenvolvimento das pesquisas espaciais tem resultado no lançamento de uma grande variedade de satélites, os quais são capazes proporcionar imagens em diferentes resoluções espaciais, temporais, espectrais e radiométricas. De acordo com Bello (2014), a importância do Sensoriamento Remoto em estudos sobre desastres ambientais está relacionada à capacidade de aquisição de dados de maneira rápida antes e após o evento, permitindo assim avaliar de forma temporal os danos causados e auxiliar na elaboração de diretrizes de atuação em uma fase posterior.

Nos estudos de Sensoriamento Remoto, a classificação multitemporal e detecção de mudanças tornouse um campo ativo, devido à crescente disponibilidade de séries temporais de imagens e ao interesse de monitorar as mudanças da Terra em escalas local e global (CAMPS-VALLS et al., 2011). Detecção de Mudança corresponde a uma classe de técnicas direcionadas à análise de duas ou mais imagens adquiridas em mesmo local geográfico em instantes distintos (SINGH, 1989). Resultados proporcionados por tais técnicas podem apoiar diversas aplicações, por exemplo, os estudos das dinâmicas de uso e cobertura do solo, monitoramento ecológico, avaliação de parâmetros do solo e desastres ambientais (ELAGOUZ et al., 2019; PANGALI SHARMA et al., 2019; WILLIS, 2015; ZHU et al., 2019).

Adicionalmente, as técnicas de detecção de mudança podem ser divididas em duas categorias: supervisionadas e não supervisionadas. A denominação supervisionada ou não é derivada das abordagens de aprendizado de máquina, usualmente empregadas em Classificação de Imagens.

Recentemente, o Brasil tem enfrentado uma série de desastres que ocasionaram impactos imensuráveis ao meio ambiente e sua biodiversidade, assim como danos sociais e econômicos. No ano de 2015, em Mariana, estado de Minas Gerais, ocorreu o rompimento da barragem Fundão, que comportava os rejeitos gerados pela intensa atividade de mineração local. O rompimento provocou uma onda de lama e detritos de aproximadamente 34 milhões de metros cúbicos (SAMARCO, 2016), causando mortes, assoreamento dos caminhos de drenagem e destruição em municípios a jusante do local de rompimento.

No início de 2019, outro desastre relacionado a rompimento de barragem provocou danos sociais ainda maiores que o evento ocorrido em Mariana. Situada em Brumadinho, Minas Gerais, a barragem de rejeitos da mina Córrego do Feijão, da mineradora Vale S.A, despejou cerca de 13 milhões de metros cúbicos de rejeitos de mineração no meio ambiente, o que causou a morte e desaparecimento de centenas de pessoas.

A contagem de focos de incêndio que ocorreram no Brasil em 2019, entre agosto e setembro, atingiram níveis críticos quando comparado com os eventos ocorridos nos anos anteriores (SETZER et al., 2019). O bioma Cerrado foi um dos mais atingidos, com número de focos de incêndio superior à média das series históricas no mesmo período. O estado do Mato Grosso contabilizou cerca de 50 mil focos de incêndio. Dentre as várias áreas afetadas estão inclusas terras indígenas, por exemplo, a reserva Urubu Branco, com área de 168 mil hectares e população estimada de aproximadamente 580 índios Tapirapé.

Diante do atual cenário esboçado, este estudo tem como finalidade a aplicação e avaliação de técnicas 
de detecção de mudanças em imagens obtidas por Sensoriamento Remoto para o mapeamento e a análise dos desastres causados pelo rompimento das barragens em Mariana e Brumadinho, e pelas queimadas no nordeste mato-grossense. Para isso foram utilizadas imagens adquiridas pelos satélites Landsat-8, para o caso de Mariana, Sentinel-2, para o caso de Brumadinho e imagens de radar obtidas pelo Sentinel-1, para o caso das queimadas. Os métodos de detecção não supervisionada de mudanças discutidas em (CELIK, 2009; JOHNSON; KASISCHKE, 1998; MURA et al., 2008) serão empregados neste estudo.

Este artigo está organizado da seguinte forma: a Seção 2 apresenta uma breve discussão teórica sobre detecção de mudança, incluindo os métodos analisados neste estudo; na Seção 3 são tecidas discussões sobre a área de estudo, dados disponíveis e procedimentos metodológicos utilizados. Resultados e discussões referentes aos experimentos realizados serão apresentados na Seção 4; por fim, considerações finais e perspectivas futuras são discorridas na Seção 5 .

\section{MÉTODOS NÃO SUPERVISIONADOS DE DETECÇÃO DE MUDANÇA}

Segundo Camps-Valls. et al. (2011), os métodos de detecção de mudança podem ser formulados a fim de proporcionar resultados na forma de mapas binários, mapas de tipos de mudanças e mapas com classes de mudança. Em princípio, a detecção de mudanças visa à comparação de imagens multitemporais por meio de operações entre os pixels correspondentes (ILSEVER; UNSALAN, 2012). Uma forma simplista de detecção de mudança consiste na obtenção da diferença entre pares de imagens conforme a Equação 1.

$$
I_{d}(x, y)=I_{1}(x, y)-I_{2}(x, y)
$$

onde $I_{1}$ e $I_{2}$ são as imagens, compostas por uma ou mais bandas/atributos, obtidas em instantes distintos $t_{1} \mathrm{e}$ $t_{2},(x, y)$ são as coordenadas de um dado pixel nas imagens e $I_{d}$ representa a imagem que expressa as diferenças entre o par de imagens observado. No entanto, esta abordagem simplista pode sofrer forte influência de fatores como interferências atmosféricas ou mesmo variações sazonais sobre determinados alvos, exigindo assim a aplicação de métodos mais complexos.

Nos anos recentes, diferentes propostas de métodos de detecção de mudança têm sido propostas na literatura. Usualmente, tais propostas utilizaram conceitos relacionados à análise de pós-classificação, simples operações aritméticas, análise de componentes principais e filtros morfológicos (WU; DU; ZHANG, 2014).

Os métodos de detecção de mudança podem ser classificados em dois grupos: supervisionados e não supervisionados. No primeiro caso, a detecção é realizada por meio de comparações de mapeamentos do tipo de cobertura do solo obtidos por classificação supervisionada ou processo similar, o que faz necessário um conjunto de informações para treinamento/modelagem do método. Em contrapartida, os métodos não supervisionados realizam uma comparação e identificação de alterações entre duas ou mais imagens de forma automática (CELIK, 2009). A possibilidade de identificação de alterações temporais sem exigência de dados adicionais torna os métodos não supervisionados mais atrativos em comparação aos supervisionados.

Proposto por Malila (1980), a Análise de Vetor de Mudanças (Change Vector Analysis - CVA), consiste em uma abordagem clássica de detecção não supervisionada de mudanças. O conceito principal deste método consiste na obtenção de uma imagem de amplitude de diferenças $I_{a}$, dada por $I_{a}(x, y)=$ $\left\|I_{1}(x, y)-I_{2}(x, y)\right\|$, seguida pela distinção dos respectivos pixels segundo sua tendência em expressar ou não a ocorrência de mudança. Tal distinção é proporcionada com uso de técnicas de limiarização, como por exemplo, os algoritmos de Otsu (OTSU, 1979) ou Kittler-Illingworth (KITTLER; ILLINGWORTH, 1986). Segundo Johnson e Kasischke (1998), o método CVA é capaz de superar fragilidades de métodos de detecção supervisionada, como erros acumulativos no processo de classificação, assim como tem capacidade de processar qualquer número de atributos simultaneamente com baixo custo computacional.

Celik (2009) propõe um método de simples arquitetura computacional e que une Análise de Componentes Principais (PCA) (JOLLIFFE, 2002) e o algoritmo K-Médias (WEBB; COPSEY, 2011). Neste trabalho este método será referido como PCA-KM. De forma simplificada, este método é iniciado com o particionamento de uma imagem de amplitude de diferenças em blocos de dimensão $h \times h$ não sobrepostos, sobre os quais são construídos vetores cujas componentes são os valores internos a tais blocos. Os vetores 
extraídos são empregados na obtenção de eixos ortogonais, com uso de PCA, que define uma transformação linear sobre os vetores originais. Posteriormente, novas representações vetoriais são extraídas para cada pixel da imagem de amplitude de diferenças, a partir de blocos $h \times h$ centrados em tais pixels, e então projetados para o espaço determinado pela transformação linear revelada pela técnica PCA, considerando apenas as $S$ primeiras componentes desta projeção, o que torna $s$ um valor inteiro e limitado a $h^{2}$, mas que por convenção não ultrapassa $h$ a fim de evitar os efeitos da maldição da dimensionalidade. Por último, os dados projetados são submetidos ao algoritmo K-Médias a fim de detectar dois agrupamentos. O agrupamento mais próximo à origem do espaço projetado determina os pixels associados a não ocorrência de mudança, ao contrário do outro agrupamento, composto por pixels onde ocorrem mudanças temporais.

Mura (2008), propõe outro método de detecção de mudança com base no conceito de Filtros Sequenciais Alternantes (Alternating Sequential Filters - ASF). Analogamente aos métodos CVA e PCA-KM, inicialmente é determinada a imagem de amplitude de diferenças. Posteriormente, são aplicados ASF a fim de simplificar as diferenças expressas na imagem de diferenças e ao mesmo tempo conservar a informação geométrica dos objetos existentes. O conceito de ASF corresponde a uma sequência de operações morfológicas, onde o filtro determinado pelo elemento estruturante ira alternar entre sequências de abertura e fechamento (GONZALEZ; WOODS, 2007). A operação de abertura corresponde à aplicação de um filtro de erosão seguido pela aplicação do filtro de dilatação, já a operação de fechamento consiste na dilatação seguida pela erosão. Na proposta de Mura (2008), tais operações são aplicadas de modo iterativo e com aumento gradativo nas dimensões do elemento estruturante. O número de iterações realizadas corresponde a um parâmetro neste método. Por último, é aplicada uma limiarização sobre a filtragem alcançada. Pixel associados a valores abaixo do limiar estabelecido são caracterizados como não mudança, e valores acima deste limiar corresponde a ocorrência de mudança. Neste trabalho, este método é referenciado por ASF.

\section{MATERIAIS E MÉTODOS}

\section{1 Áreas de estudo: cenários brasileiros de recentes desastres}

Com a finalidade mapear as alterações causadas por recentes desastres ocorridos no Brasil com uso de técnicas de detecção de mudanças, foram escolhidos três eventos distintos, os quais incluem rompimento de barragens e queimadas. As áreas de estudo referentes a cada um dos desastres compreendem as regiões afetadas pelo rompimento das barragens de Mariana (Área 1) e Brumadinho (Área 2), ambas situadas no Quadrilátero Ferrífero no estado de Minas Gerais, e pelas queimadas no estado do Mato Grosso (Área 3) no entorno da reserva indígena Urubu Branco. A localização espacial destas áreas é apresentada na Figura 1.

A barragem de Fundão está inserida no contexto da bacia hidrográfica do Rio Doce e está localizada no subdistrito de Bento Rodrigues, à 35 quilômetros do município de Mariana, no estado de Minas Gerais. Na tarde de 5 de novembro de 2015 houve o rompimento desta barragem devido a uma liquefação que se iniciou na ombreira esquerda, despejando aproximadamente 34 milhões de metros cúbicos de rejeitos, que corresponde a $60 \%$ do volume em operação (SAMARCO, 2016). A "onda" de lama ficou marcada como um dos maiores desastres ambientais brasileiros afetando grande parte da Mata Atlântica, que é considerado como um dos principais hotspots da biodiversidade do planeta (CARMO et al., 2017).

A barragem do Córrego do Feijão inserida no contexto da bacia hidrográfica do Paraopeba, que é um dos importantes afluentes do rio São Francisco. O colapso da barragem provocou o despejo de aproximadamente 12 milhões de metros cúbicos de rejeitos (VALE, 2019). Esse desastre causou danos ainda mais severos que o rompimento de Mariana, sendo identificados diversos danos irreparáveis ao meio ambiente, por exemplo, a destruição de habitats e extermínio da ictiofauna ao longo da extensão da bacia atingida; o assoreamento dos corpos hídricos; e o soterramento de ecossistemas lacustres. 
Figura 1 - Localização geográfica das áreas de estudo.
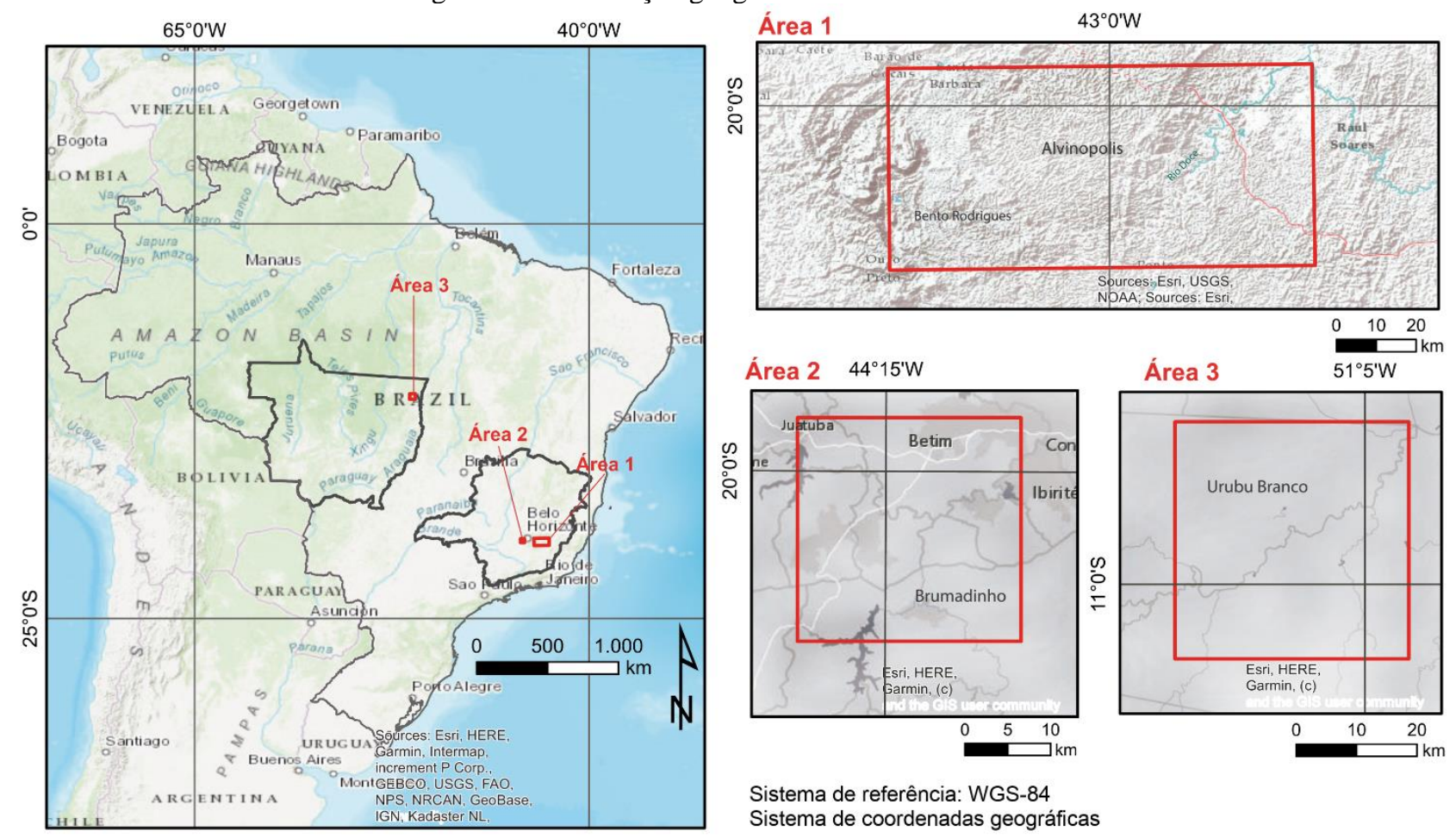

Fonte: Os autores (2020).

Durante o início do segundo semestre de 2019 houveram intensificação de focos de incêndio nas regiões do entorno da Amazônia Legal e no Centro-Oeste do país. Aproximadamente $18 \%$ dos focos de incêndio registrados no período de agosto a setembro de 2019 foram concentradas no estado do Mato Grosso. Neste mesmo período, o bioma do cerrado teve aproximadamente 95 mil quilômetros quadrados de área queimada (SETZER et al., 2019). Frente a este acontecimento, foi selecionada como área de estudo uma região situada no estado do Mato Grosso centrada pelo rio Tapirapé. Este rio separa a área de estudo em uma porção superior, que compreende as terras indígenas denominadas por Urubu Branco, e a porção inferior que contém uma porção do município de Luciara.

\subsection{Dados utilizados}

Com finalidade de verificar as mudanças causadas pelo rompimento da barragem de Mariana (Área 1), foram empregadas imagens obtidas pelo satélite Landsat-8, através do sensor multiespectral OLI (Operational Land Imager), nas datas 25 de setembro de 2015 e 10 de agosto de 2016, abrangendo assim um período anterior e posterior ao desastre ocorrido. Tais imagens possuem resolução espacial de 30 metros e foram consideradas seis bandas espectrais, localizadas no intervalo do azul ao infravermelho de ondas curtas. A Figura 2 apresenta as imagens utilizadas e limitadas à área de estudo.

Com relação ao evento ocorrido na região de Brumadinho (Área 2), foram empregadas imagens obtidas pelo satélite Sentinel-2, compostas por quatro bandas espectrais (azul, verde, vermelho e infravermelho) e resolução espacial de 10 metros. As datas de imageamento ocorreram em 22 de janeiro e $1^{\circ}$ de fevereiro de 2019. A Figura 3 ilustra tais imagens.

Por último, as imagens adotadas sobre a região de Urubu Branco foram obtidas pelo radar de abertura sintética a bordo do satélite Sentinel-1. Este sensor utiliza ondas com comprimento na banda C e proporciona imagens resolução espacial de aproximadamente 10 metros segundo as combinações de polarização HH e HV. As aquisições das imagens são datadas em 5 de junho e 21 de setembro de 2019, as quais são ilustradas na Figura 4. 
Figura 2 - Na porção superior, imagens do satélite Landsat-8 referentes à área de estudo da barragem de Mariana (Área 1), em composição natural (banda/canal - 4/R, 3/G, 2/B). Na parte inferior, amostras de verdade terrestre sobre regiões de mudança e não mudança, utilizadas para o cálculo de acurácia nos experimentos que seguem.
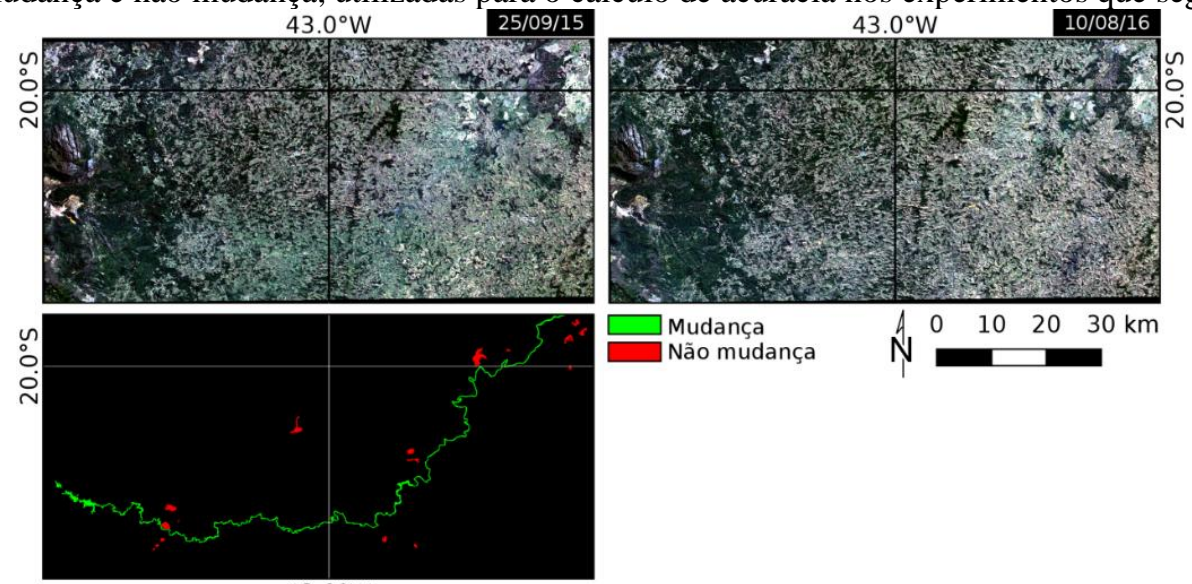

$43.0^{\circ} \mathrm{W}$

Fonte: Os autores (2020).

Figura 3 - Na parte esquerda e central, imagens do satelite Sentinel-2, referentes à área de estudo da barragem de

Brumadinho (Área 2), em composição natural (banda/canal - 4/R, 3/G, 2/B). Ao lado direito, amostras de verdade terrestre sobre regiões de mudança e não mudança, utilizadas para o cálculo de acurácia nos experimentos que seguem.

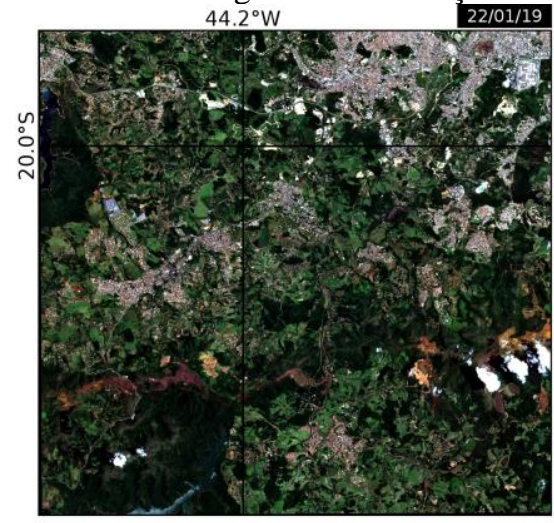

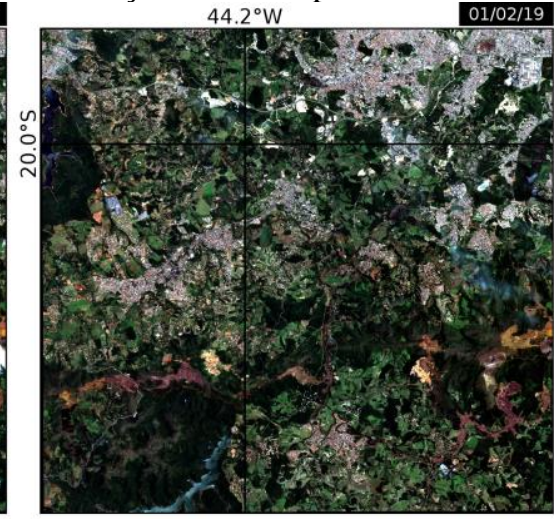

Fonte: Os autores (2020).

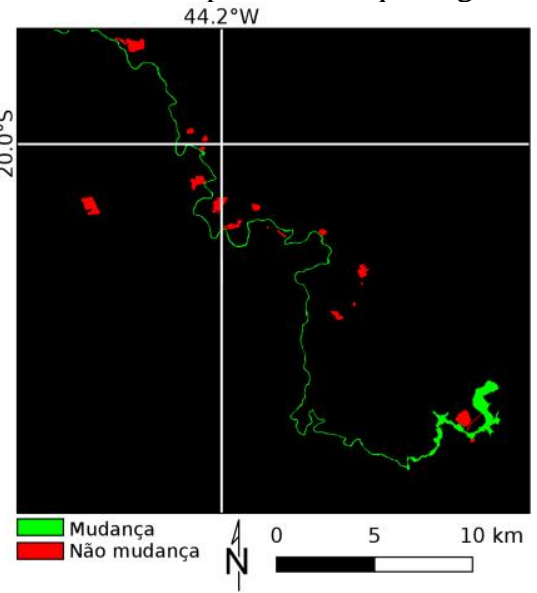

N

Com finalidade de avaliar a acurácia dos mapas de mudança que serão gerados através da aplicação dos métodos descritos na Seção 2, foram coletadas amostras correspondentes às mudanças ocorridas em cada área de estudo. Na Tabela 1 são exibidas as dimensões das amostras coletadas bem como nas Figuras 2, 3 e 4 a distribuição espacial das mesmas.

Tabela 1 - Amostras de validação referentes a cada uma das áreas de estudo.

\begin{tabular}{c|c|c|c|c}
\hline Local & Tipo & $\mathbf{N}^{\circ}$ Pixels & Área Total $\left(\mathbf{k m}^{2}\right)$ & \% da Imagem \\
\hline \multirow{2}{*}{ Área 1 } & Mudança & 38457 & 34,6 & $0,69 \%$ \\
\cline { 2 - 5 } & Não Mudança & 63194 & 56,9 & $1,13 \%$ \\
\hline \multirow{2}{*}{ Área 2 } & Mudança & 56000 & 5,6 & $1,00 \%$ \\
\cline { 2 - 5 } & Não Mudança & 44500 & 4,45 & $0,80 \%$ \\
\hline \multirow{2}{*}{ Área 3 } & Mudança & 38457 & 15,5 & $0,69 \%$ \\
\cline { 2 - 5 } & Não Mudança & 63194 & 15,5 & $1,13 \%$ \\
\hline
\end{tabular}

Fonte: Os autores (2020). 
Figura 4 - Na parte esquerda e central, imagens referentes a área de queimada no Mato Grosso (Área 3), em composição colorida (polarização/canal - HH/R, HV/G, VV/B), referentes ao radar Sentinel-1. Ao lado direito, amostras de verdade terrestre sobre regiões de mudança e não mudança, utilizadas para o cálculo de acurácia nos

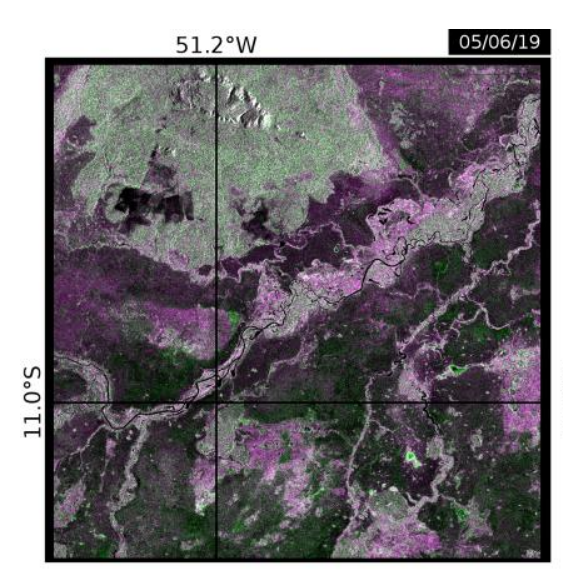
experimentos que seguem

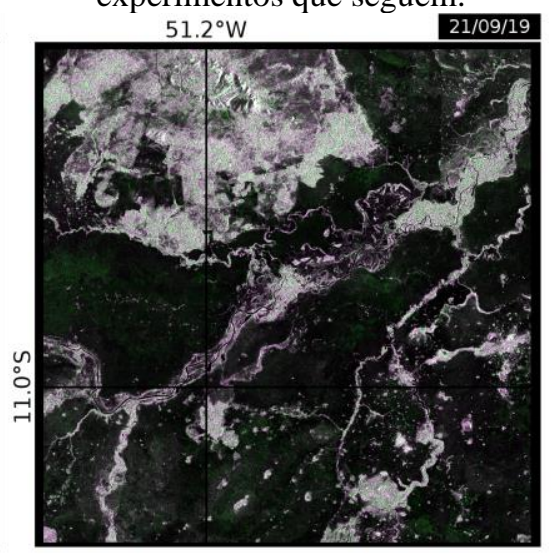

Fonte: Os autores (2020).

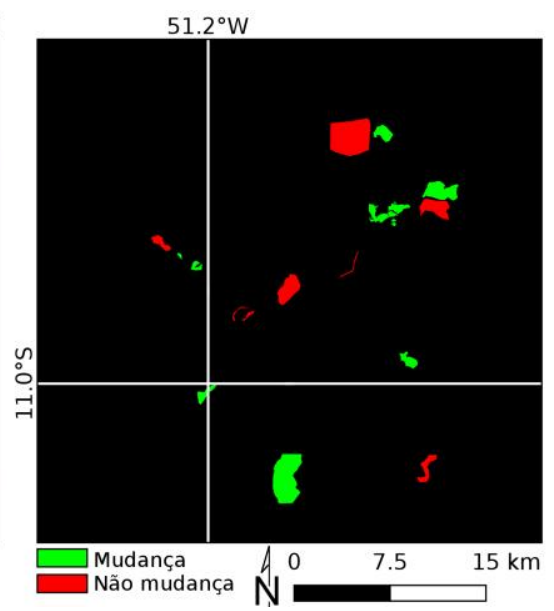

\subsection{Procedimentos metodológicos}

As etapas metodológicas que abrangem este estudo são estruturadas segundo o diagrama da Figura 5. Esse diagrama resume as etapas de obtenção de dados, as etapas de processamento e por fim a avaliação dos resultados.

Com relação à primeira área de estudo, partindo do par de imagens obtidos pelo sensor OLI, são efetuadas diferentes aplicações dos métodos CVA e PCA-KM diante diferentes configurações de parâmetros associados e atributos espectrais. O parâmetro relacionado ao método CVA consiste na técnica de limiarização adotada, dentre os algoritmos de Otsu e Kittler-Illinghworth. De modo distinto, os parâmetros do método PCAKM correspondem às possíveis combinações envolvendo os valores de $s \leq h \in\{3,5,7\}$. O limite imposto para tais parâmetros decorre da altíssima demanda computacional exigida para valores de $h$ superiores a 7 (i.e., o próximo valor válido para $h$ após 7 é o 9 , que caso utilizado determinaria uma vizinhança $9 \times 9$, que por sua vez se desdobra em um espaço de atributos estendido de 81 componentes, sobre o qual são consideradas as $S$ primeiras componentes principais - vide Seção 2).

Figura 5 - Diagrama sequencial de procedimentos envolvidos na detecção de mudanças.

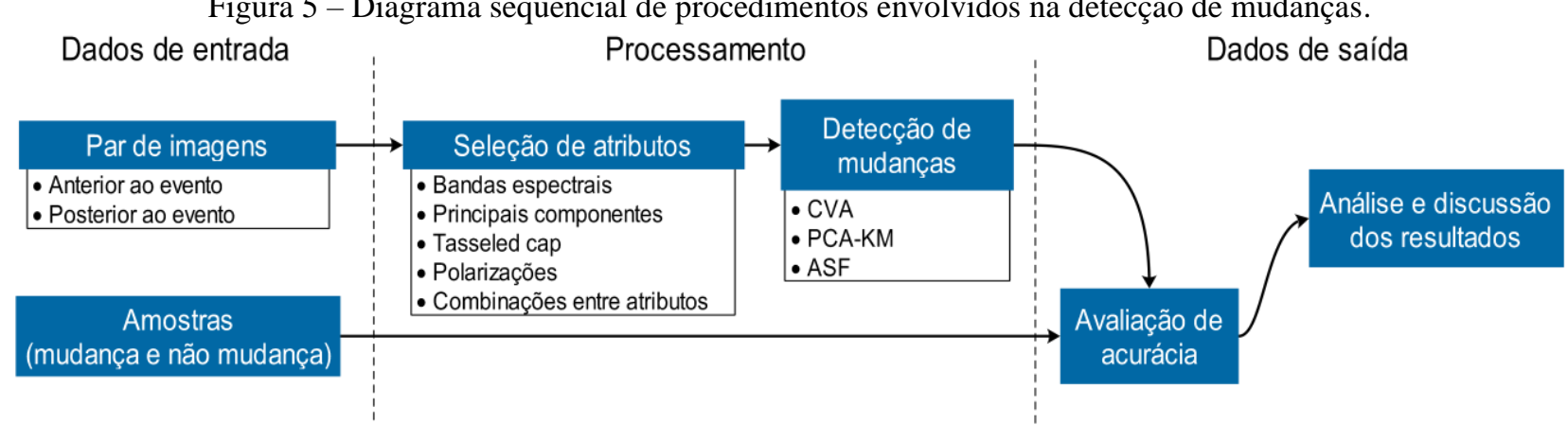

Fonte: Os autores (2020).

Além das configurações de parâmetros citadas, os métodos adotados neste experimento foram aplicados sobre: (i) as bandas espectrais compreendidas entre o intervalo do azul ao infravermelho de ondas curtas, denotadas por "Bandas-6"; (ii) três primeiras componentes principais extraídas da projeção das seis bandas espectrais compreendidas entre o intervalo do azul ao infravermelho de ondas curtas, denotadas por "PCA-1 3"; e (iii) atributos obtidos pela técnica Tasseled Cap (CRIST, 1985), denotado por "TTC".

De modo análogo, para a área de Brumadinho, o par de imagens obtidas pelo satélite Sentinel-2 será submetido aos métodos CVA e PCA-KM. As mesmas configurações de parâmetros estipuladas para uso sobre os dados referentes à área de Mariana foram também estabelecidas para este experimento. Por outro lado, a 
respeito dos atributos utilizados no processo de detecção de mudança, foram consideradas: (i) as bandas espectrais compreendidas entre o intervalo do azul ao infravermelho próximo, denotado por "Bandas-4"; e (ii) as três primeiras componentes principais extraídas destas bandas, novamente denotado por "PCA-1 3".

Por último, referente à região da reserva indígena Urubu Branco, os métodos CVA e ASF serão aplicados às imagens obtidas pelo satélite Sentinel-1. A fim de reduzir os efeitos do ruído speckle, as polarizações $\mathrm{HH}$ e $\mathrm{HV}$ foram submetidas ao filtro passa baixa com núcleo $5 \times 5$. Os parâmetros utilizados pelo método CVA serão análogos aos aplicados nos casos anteriores. O número de iterações executadas pelo método ASF será verificada de 1 a 4.

Cabe observar que o método CVA foi empregado nas três áreas de estudo, uma vez que este é um método amplamente conhecido e utilizado na literatura. Por outro lado, o método PCA-KM foi adotado sobre dados ópticos (i.e., obtidas via sensor OLI e satélite Sentinel-2), uma vez que é indicado para imagens desta natureza. Pela mesma razão, o método ASF foi aplicado somente aos dados obtidos por radar, através do satélite Sentinel-1.

Os resultados a serem obtidos em cada área de estudo, diante cada configuração de parâmetro e atributos considerados, serão avaliados diante a medida F1-Score (SASAKI, 2007), computada com base nas amostras, de mudança e não mudança, identificadas (Figuras 2, 3 e 4). Por sua vez, os resultados de maior acurácia, para cada área de estudo, serão selecionados para análise e discussão das mudanças ocorridas.

As implementações dos métodos utilizados neste estudo foram realizadas com uso da linguagem de programação IDL. O software ENVI foi empregado para o redimensionamento das imagens à porção das áreas de estudo e coleta de amostras de verdade terrestre. O sistema QGIS foi adotado para geração de mapas e demais representações munidas de informações cartográficas.

\section{RESULTADOS E DISCUSSÃO}

De posse das imagens e respectivas amostras de mudança e não mudança, e seguindo os procedimentos metodológicos delimitados na Seção 3.3, foram obtidos diferentes mapas de detecção de mudança. Para cada área e suas respectivas configurações de teste foram obtidos os valores de F1-Score, conforme ilustra a Figura 6.

Com relação aos resultados obtidos em relação à Área 1, é verificado que o maior valor de F1-Score foi 0,756 , alcançado pelo método PCA-KM com uso de seis bandas espectrais como atributos (i.e., "Bandas6"). Por outro lado, o mais baixo nível de acurácia foi de 0,701 , proporcionado pelo método CVA com uso dos atributos PCA-1 3 e TTC. Observa-se ainda que o método PCA-KM proporcionou diferentes valores médios de acurácia quando utilizados atributos distintos, ao contrário do método CVA, mostrando-se menos sensível aos atributos de entrada deste caso

Com base em uma análise qualitativa dos mapas de mudança da Área 1, foi observado que o método CVA proporcionou melhores resultados quando associado ao algoritmo de Otsu, já os limiares de estabelecidos pelo algoritmo de Kittler-Illingworth dificultaram a visualização das áreas afetadas pelo desastre por capturar um maior número de mudanças de forma errônea.

O método PCA-KM desempenhou resultados mais consistentes na detecção de mudanças, porém, também foi prejudicado pela diferença de intensidade das imagens e aglomerações de mudanças marginais conforme a variação do parâmetro $h$. A grande detecção de falsos positivos pode ser associada a presença de nuvens no momento da aquisição; cuja ocorrência implica na alteração do contraste da imagem (i.e., espalhamentos Rayleigh e Mie) de forma não homogênea.

Ainda com relação ao método PCA-KM, limitado a configuração de parâmetros $s, h=7$, ao comparar os resultados alcançados com atributos "Bandas-6" e "PCA-1 3", cujos valores de F1-Score são respectivamente 0,756 e 0,729 , verifica-se que o primeiro conjunto de atributos proporciona excesso de falsos positivos, ao contrário do segundo conjunto que evitou detecções de mudanças em função de variações de brilho/contraste. A Figura 7 ilustra estes dois casos discutidos. 
Figura 6 - Valores de F1-Score, distintos por áreas de estudo, método de detecção de mudança e os respectivos testes utilizados.

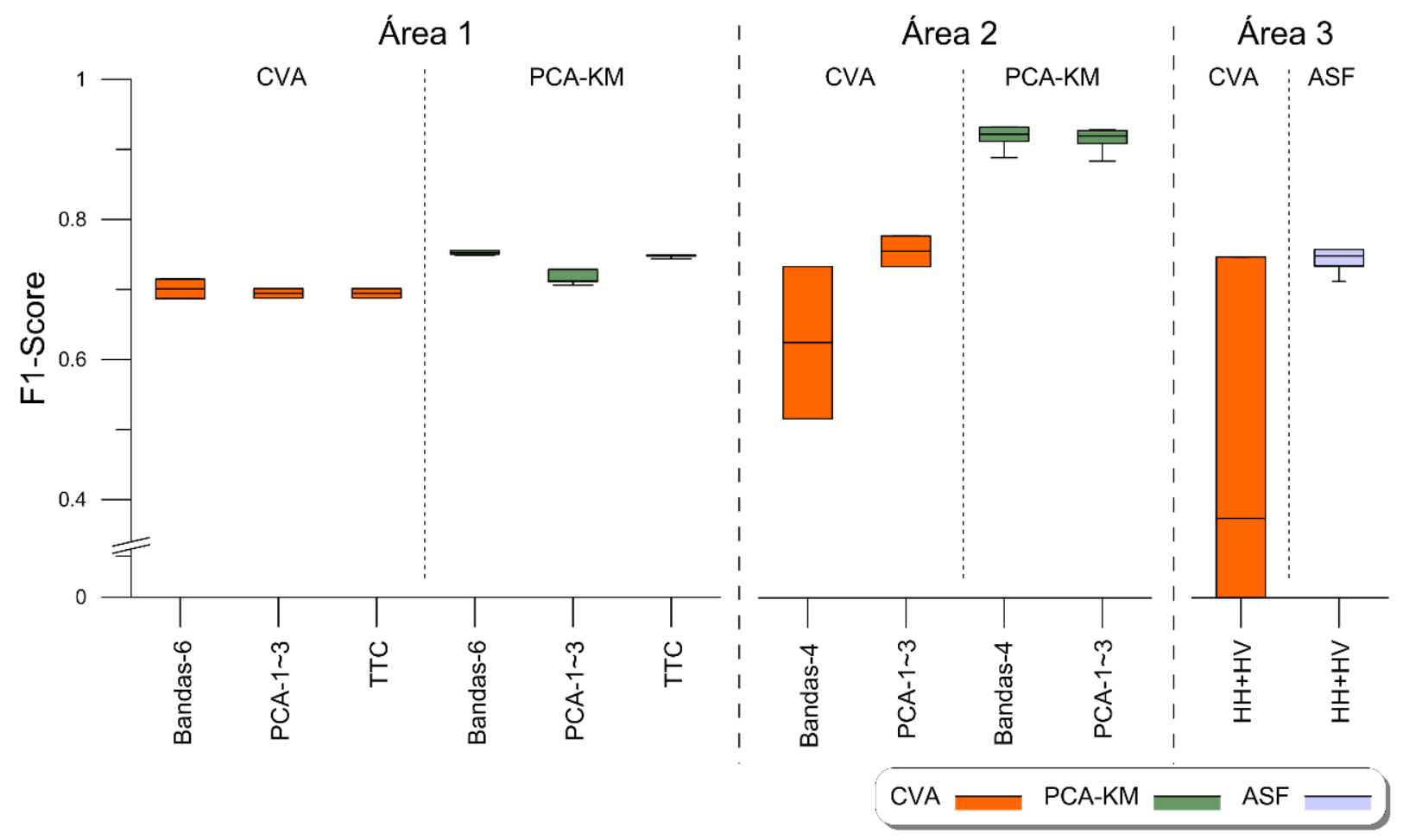

Fonte: Os autores (2020).

Com relação a segunda área de estudo, é verificada mais uma vez a superioridade do método PCAKM em comparação ao CVA, independentemente de atributos e configuração de parâmetros utilizados. O melhor resultado obtido por este método, associado aos atributos "Bandas-4" e parâmetros $s, h=7$, apontou valor de F1-Score igual a 0,932. O método CVA proporcionou resultado de maior acurácia com F1-Score de 0,865 , sendo este $7,8 \%$ menor que resultado alcançado pelo PCA-KM.

Figura 7 - Resultados potenciais alcançados pelo método PCA-KM sobre a área de estudo compreendida pelas abrangências da barragem de Mariana (Área 1).

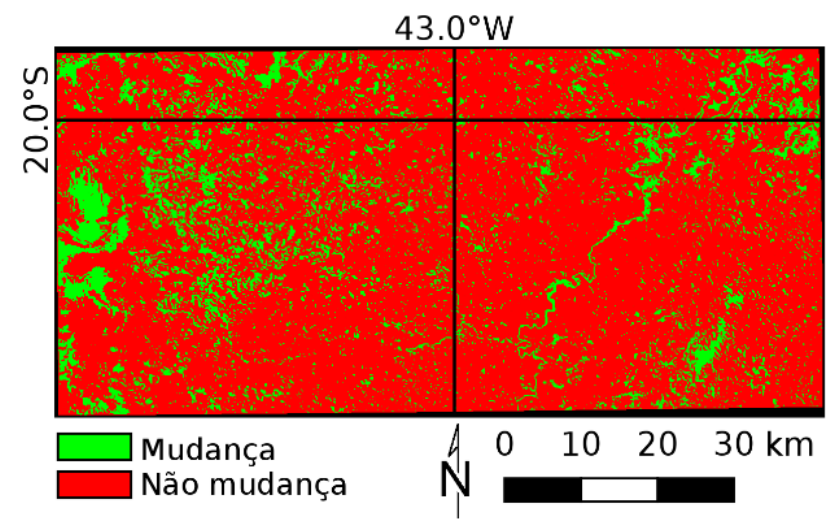

(a) PCA-1 3-Parâmetros $s, h=7$

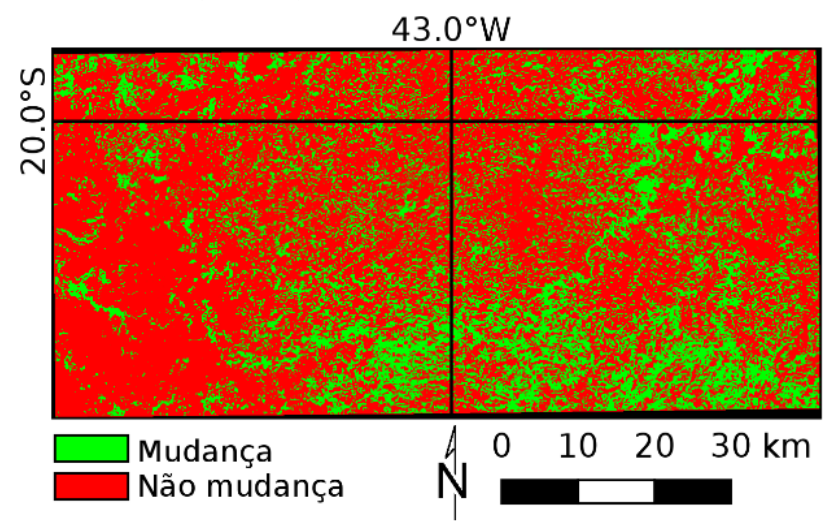

(b) Bandas-6 - Parâmetros $s, h=7$

Fonte: Os autores (2020).

De modo geral, o método CVA não gerou resultados adequados quando utilizado o algoritmo de limiarização de Kittler-Illinghworth, ao contrário de quando adotado o algoritmo de Otsu e atributos "PCA1 3". Avaliando os dados estatísticos (Figura 6), fica evidente que a diferença interquartil aponta que este o método não mostrou bom desempenho com atributos "Bandas-4". Por consistir em um método mais simples de detecção de mudanças, seus resultados tenderam a ser inferiores aos observados no método KM-PCA. Ainda, a quantidade de bandas disponíveis pode ser apontada como fator associado ao baixo desempenho do método CVA. 
Segundo uma análise visual dos resultados gerados, observa-se que o método PCA-KM foi capaz usufruir de sua maior complexidade algorítmica para identificação de áreas de mudanças, conforme ilustra a Figura 8. Mesmo com o número de bandas reduzidas, as transformações embutidas em seu algoritmo foram capazes de ressaltar as principais diferenças que existiam na imagem. Os resultados alcançados com os atributos "Bandas-6" e "PCA-1 3" são similares, exceto pelo fato de que o teste "PCA-1 3" sofreu maior influência de efeitos de reflexo e espalhamento da luz dos centros urbanos, captando assim alguns falsos positivos, conforme as marcações indicadas no mapa de mudanças na Figura 8. Em relação à detecção do desastre, ambos os atributos foram capazes de identificar, em sua maioria, as áreas afetadas.

É razoável admitir que os desastres ambientais devam ser identificados de forma rápida e eficaz, viabilizando assim o estudo e monitoramento adequado das regiões afetadas. Tomadas de decisões tardias podem causar uma série de problemas sociais, econômicos e ambientais. Desta forma, é possível observar a partir da comparação dos resultados referentes às Áreas 1 e 2, que os sensores óticos estão sob uma forte influência das condições atmosféricas e a presença das nuvens pode interferir de forma crucial na capacidade de detecção das alterações causadas pelo desastre. Neste estudo, em particular sobre a Área 1, imagens de livre acesso e capazes de representar a situação "pós-desastres" com quantidades de cobertura de nuvem inferiores a 5\% só foram possíveis após aproximadamente um ano ocorrido o evento. Por outro lado, os fatores atmosféricos não inviabilizam o uso de dados ópticos, como mostrado nos experimentos sobre a Área 2. Neste caso, as altas taxas de revisita (5 dias) associada a alta resolução espacial possibilitaram melhores resultados de detecção de mudança.

Diferente dos casos anteriores, os experimentos sobre a terceira área de estudo contempla a obtenção de mapas de mudança com uso de imagens adquiridas por sensor radar de abertura sintética, a bordo do satélite Sentinel-1. Neste caso, o experimento é limitado a apenas dois testes devido à disponibilidade de apenas duas polarizações (i.e., HH e HV). Foi verificado que o método ASF proporcionou o resultado de maior acurácia em sua primeira iteração, com F1-Score de 0,75 . Ao passo que o número de iterações aumenta, são fornecidos mapas com menor presença de pixels isolados (i.e., mapas mais suavizados) e com menor capacidade de distinguir mudanças locais. O método CVA alcançou maior valor de F1-Score em 0,74 quando feito uso do algoritmo de Kittler-Illinghworth; sendo este similar ao ASF com uma iteração. Por outro lado, quando utilizado o algoritmo de Otsu, os resultados obtidos foram inviáveis.

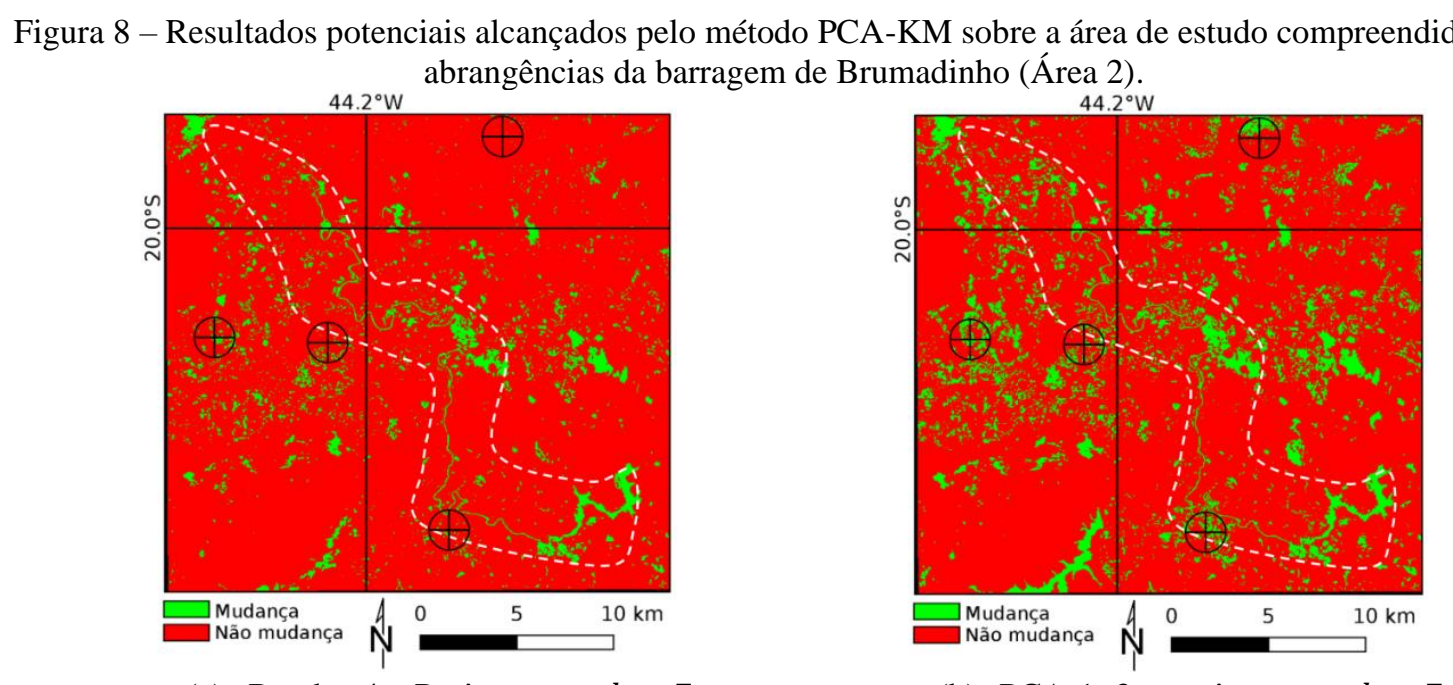

(a) Bandas-4 - Parâmetros $s, h=7$

(b) PCA-1 3-parâmetros $s, h=7$

Fonte: Os autores (2020).

O método CVA utilizando os limiares pela técnica Otsu tiveram de ser descartados devido a incapacidade de detectar qualquer mudança na imagem. Já para o caso em que foi utilizada a limiarização de Kittler-Illinghworth, as mudanças puderam ser identificadas de forma bem próxima a primeira iteração realizada pelo método ASF. Devido a imagem de radar possuir muito ruído, o filtro aplicado durante o préprocessamento talvez não tenha sido eficiente melhores resultados do método CVA. Já para o ASF o filtro foi suficiente para detectar as variações de textura e perda de vegetação da região. 
As ocorrências de mudança apresentadas na Figura 9 são compatíveis com as regiões de focos de incêndio previamente observadas em Setzer et. al (2019). Os resultados obtidos neste experimento permitem destacar que as imagens de radar surgem como opção potencial para identificação de perda de vegetação por incêndios, uma vez que essas imagens possuem uma alta resolução espacial e não são influenciadas por fatores atmosféricos.

Figura 9 - Resultados potenciais alcançados pelo método PCA-KM sobre a área de estudo compreendida pelas

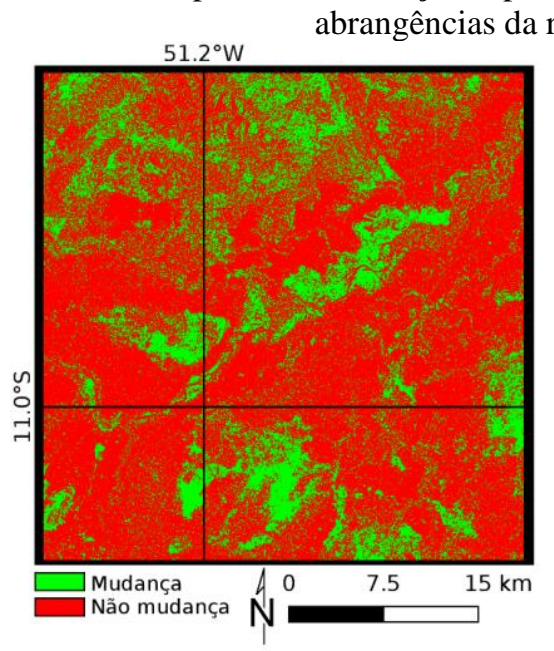

(a) ASF - uma iteração

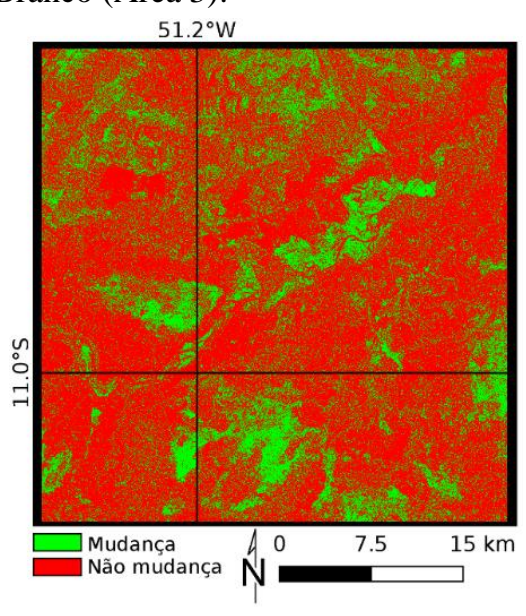

(c) CVA - Kittler-Illinghworth

Fonte: Os autores (2020)

\section{CONCLUSÃO}

A detecção não supervisionada de mudanças aponta como importante instrumento em estudos relacionados a desastres ambientais. Este trabalho abordou o mapeamento de três desastres ambientais ocorridos no Brasil entre os anos de 2015 a 2019 por meio da aplicação de métodos de detecção não supervisionadas.

Admitindo uma avaliação qualitativa sobre os mapeamentos obtidos, os resultados alcançados com uso de imagens ópticas de alta resolução espacial e temporal, adquiridas pelo satélite Sentinel-2, foram superiores aos resultados obtidos pelos mesmos métodos em uma problemática similar, porém, com dados de menor resolução espacial e temporal. Quanto aos métodos utilizados nestes experimentos, PCA-KM demonstrou superioridade. No entanto, trata-se de um método mais sofisticado em comparação a abordagem não supervisionada clássica, representada pelo método CVA.

Em relação aos resultados obtidos para o mapeamento de áreas afetadas por queimada com uso de imagens de radar, verifica-se que devido a forma de imageamento coerente realizado por sensores radar, os mapas de mudança gerados são afetados com a presença de ruídos, caracterizado pela presença de pixels isolados. No entanto, este fator foi minimizado quando utilizado o método mais apropriado, no caso o ASF, sendo este capaz de eliminar ruídos excessivos e destacar em grande maioria as áreas que sofreram alteração. Neste contexto, o método ASF podendo esta ser considerado uma ferramenta importante nos estudos de perda de biomassa por queimadas.

Como perspectivas futuras para este estudo, são destacadas: (i) estender as comparações realizadas com outros métodos não supervisionados propostos na literatura; (ii) realizar comparações com métodos supervisionados de detecção de mudança; (iii) desenvolver estratégias ou aprimorar os métodos existentes a fim de lidar com mudanças abruptas em regiões de borda, causadas principalmente pela diferença de intensidade dos pixels, e evitar por sua vez a ocorrência de falsos positivos.

\section{Agradecimentos}

Os autores agradecem a FAPESP (Proc. 2018/01033-3) pelo apoio financeiro. 


\section{Contribuição dos Autores}

L.V. Oliveira, R. G. Negri e L. B. L. Santos atuaram conjuntamente na conceptualização, investigação, metodologia, validação, visualização, revisão e edição; L.V. Oliveira foi responsável pela curadoria dos dados; R. G. Negri e L. B. L. Santos executaram supervisão; L.V. Oliveira e R. G. Negri atuaram na análise formal, desenvolvimento de software; R. G. Negri foi responsável pela aquisição de financiamento e administração do projeto.

\section{Conflitos de Interesse}

Os autores declaram que não há conflitos de interesse.

\section{Referências}

ADIKARAM, K. K. N. B.; NAWARATHNA, C. M. Business sector preparedness in disaster management: Case study with businesses in Southern Sri Lanka in both aspects of natural and technological disasters. Procedia Engineering, v. 212, p. 918-920, 2018. Elsevier Ltd. DOI: 10.1016/j.proeng.2018.01.118.

BELLO, O. M.; AINA, Y. A. Satellite Remote Sensing as a Tool in Disaster Management and Sustainable Development: Towards a Synergistic Approach. Procedia - Social and Behavioral Sciences, v. 120, p. 365-373, 2014. Elsevier DOI: 10.1016/j.sbspro.2014.02.114.

CAMPS-VALLS, G.; TUIA, D.; GÓMEZ-CHOVA, L.; JIMÉNEZ, S.; MALO, J. Remote Sensing Image Processing. Synthesis Lectures on Image, Video, and Multimedia Processing, v. 5, n. 1, p. 1-192, 2011. Morgan \& Claypool Publishers LLC DOI: 10.2200/s00392ed1v01y201107ivm012.

CARMO, F. F. DO; KAMINO, L. H. Y.; JUNIOR, R. T.; et al. Fundão tailings dam failures: the environment tragedy of the largest technological disaster of Brazilian mining in global context. Perspectives in Ecology and Conservation, v. 15, n. 3, p. 145-151, 2017. DOI: 10.1016/j.pecon.2017.06.002.

CELIK, T. Unsupervised change detection in satellite images using principal component analysis and $\kappa$-means clustering. IEEE Geoscience and Remote Sensing Letters, 2009. DOI: 10.1109/LGRS.2009.2025059.

CRIST, E. P. A TM Tasseled Cap equivalent transformation for reflectance factor data. Remote Sensing of Environment, 1985. DOI: 10.1016/0034-4257(85)90102-6.

ELAGOUZ, M. H.; ABOU-SHLEEL, S. M.; BELAL, A. A.; EL-MOHANDES, M. A. O. Detection of land use/cover change in Egyptian Nile Delta using remote sensing. Egyptian Journal of Remote Sensing and Space Science, 2019. Elsevier B.V. DOI: 10.1016/j.ejrs.2018.10.004.

GONZALEZ, R. C.; WOODS, R. E. Digital Image Processing. 3a ed. Pearson, 2007.

GRUBER, T.; WILLBERG, M. Signal and error assessment of GOCE-based high resolution gravity field models. Journal of Geodetic Science, v. 9, n. 1, p. 71-86, 2019. DOI: 10.1515/jogs-2019-0008.x

HIDAlgO, J.; BAEZ, A. A. Natural Disasters. Critical Care Clinics, 2019. W.B. Saunders DOI: 10.1016/j.ccc.2019.05.001.

İLSEVER, M.; UNSALAN, C. Two-Dimensional Change Detection Methods. 2012 ${ }^{\circ}$ ed. Springer-Verlag London, 2012.

JOHNSON, R. D.; KASISCHKE, E. S. Change vector analysis: A technique for the multispectral monitoring of land cover and condition. International Journal of Remote Sensing, v. 19, n. 3, p. 411-426, 1998. Informa \{UK\} Limited DOI: 10.1080/014311698216062.

JOLLIFFE, I. T. Principal Component Analysis. 2a ed. New York: Springer-Verlag, 2002.

KITTLER, J.; ILLINGWORTH, J. Minimum error thresholding. Pattern Recognition, v. 19, n. 1, p. 41-47, 1986. DOI: 10.1016/0031-3203(86)90030-0.

MALILA, W. A. Change Vector Analysis: an Approach for Detecting Forest Changes With Landsat. Proceedings of the Society of Photo-Optical Instrumentation Engineers, p. 326-336, 1980.

MURA, M. D.; BENEDIKTSSON, J. A.; BOVOLO, F.; BRUZZONE, L. An unsupervised technique based on morphological filters for change detection in very high resolution images. IEEE Geoscience and Remote Sensing Letters, v. 5, n. 3, p. 433-437, 2008. DOI: 10.1109/LGRS.2008.917726.

OTSU, N. Otsu 1979 Otsu thresold method. IEEE Transactions on Systems, Man, and Cybernetics, v. 9, 
n. 1, p. 62-66, 1979. DOI: 10.1109/TSMC.1979.4310076.

PANGALI SHARMA, T. P.; ZHANG, J.; KOJU, U. A.; et al. Review of flood disaster studies in Nepal: A remote sensing perspective. International Journal of Disaster Risk Reduction, v. 34, p. 18-27, 2019. Elsevier Ltd DOI: 10.1016/j.ijdrr.2018.11.022.

POURSANIDIS, D.; CHRYSOULAKIS, N. Remote Sensing, natural hazards and the contribution of ESA Sentinels missions. Remote Sensing Applications: Society and Environment, v. 6, p. 25-38, 2017. Elsevier DOI: 10.1016/j.rsase.2017.02.001.

SAMARCO. Balanço de ações: Um ano depois do rompimento de Fundão. Minas Gerais, 2016.

SASAKI, Y. The truth of the F-measure. Teach Tutor mater, 2007.

SETZER, A. W.; MORELLI, F.; MARTINS, G.; ROMÃO, M. Infoqueima Boletim Mensal de Monitoramento de Queimadas Editores: Colaboradores: Editoração: Endereço para Correspondência: INFOQUEIMA. 2019.

SINGH, A. Review Articlel: Digital change detection techniques using remotely-sensed data. International Journal of Remote Sensing, v. 10, n. 6, p. 989-1003, 1989. Taylor \& Francis DOI: 10.1080/01431168908903939.

TOMINAGA, L. K.; SANTORO, J.; AMARAL, R. Desastres Naturais : conhecer para prevenir. 3a ed. São Paulo: Instituto geológico, 2015.

VALE. Esclarecimentos sobre a Barragem I da Mina de Córrego do Feijão. Disponível em: $<$ http://brumadinho.vale.com/Esclarecimentos-sobre-a-barragem-I-da-Mina-de-Corrego-dofeijao.html>. Acesso em: 11/10/2019.

WANDERLEY, L. J.; MANSUR, M. S.; MILANEZ, B.; PINTO, R. G. Desastre da Samarco/Vale/BHP no Vale do Rio Doce: aspectos econômicos , políticos e socio ambientais. Ciência e Cultura, v. 68, n. 3, p. 30-35, 2016. FapUNIFESP (SciELO) DOI: 10.21800/2317-66602016000300011.

WEBB, A. R.; COPSEY, K. D. Statistical Pattern Recognition: Third Edition. 2011.

WILLIS, K. S. Remote sensing change detection for ecological monitoring in United States protected areas. Biological Conservation, v. 182, p. 233-242, 2015. Elsevier Ltd DOI: 10.1016/j.biocon.2014.12.006.

WU, C.; DU, B.; ZHANG, L. Slow feature analysis for change detection in multispectral imagery. IEEE Transactions on Geoscience and Remote Sensing, 2014. DOI: 10.1109/TGRS.2013.2266673.

ZHU, L.; WALKER, J. P.; YE, N.; RÜDIGER, C. Roughness and vegetation change detection: A preprocessing for soil moisture retrieval from multi-temporal SAR imagery. Remote Sensing of Environment, v. 225, p. 93-106, 2019. Elsevier Inc. DOI: 10.1016/j.rse.2019.02.027.

\section{Biografia do autor principal}

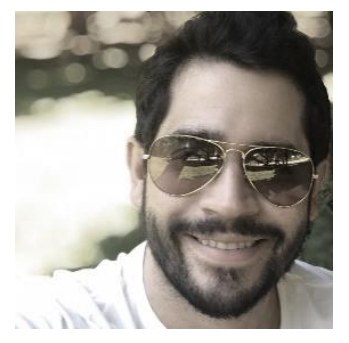

Lucas Valerio de Oliveira, nascido em São Paulo na data 01/02/1992. Graduado em Engenharia Ambiental pela Universidade Estadual Paulista - Instituto de Ciência e Tecnologia - São José dos Campos. Atuação em pesquisas relacionadas à modelagem de bacias hidrográficas, desastres naturais e detecção de mudança por uso de Sensoriamento Remoto e algoritmos computacionais. 